\title{
Ensino de Design Visual de Aplicativos Móveis no Ensino Fundamental
}

\author{
Miriam Nathalie Fortuna Ferreira ${ }^{\dagger}$ \\ Departamento de Expressão gráfica \\ Universidade Federal de Santa Catarina \\ (UFSC) - SC, Brasil \\ nathalie.fortuna@prosgrad.ufsc.br
}

\author{
Christiane Gresse von Wangenheim \\ Departmento de Informática e \\ Estatística \\ Universidade Federal de Santa Catarina \\ (UFSC) - SC, Brasil \\ c.wangenheim@ufsc.br
}

\author{
Berenice Santos Gonçalves \\ Departamento de Expressão gráfica \\ Universidade Federal de Santa Catarina \\ (UFSC) - SC, Brasil \\ berenice@cce.ufsc.br
}

\author{
Jean Carlo Rossa Hauck \\ Departmento de Informática e \\ Estatística Universidade Federal de \\ Santa Catarina (UFSC) - SC, Brasil \\ jean.hauck@ufsc.br
}

\author{
Giselle Araújo e Silva de Medeiros \\ Programa de Pós-Graduação em \\ Educação - Universidade Federal de \\ Santa Catarina (UFSC) - SC, Brasil \\ giselle.medeiros@posgrad.ufsc.br
}

\begin{abstract}
Computing in $\mathrm{K}-12$ is typically taught through practical programming activities in which students create software artifacts such as mobile applications. This approach, however, may not cover other important competencies such as user interface design, which are essential for software development. Within this context, this article presents an instructional unit that incorporates the teaching of UI design competencies into computing education. The instructional unit was developed in a systematic way following an instructional design process, and applied and evaluated in a Brazilian public middle school. First results indicate that dynamics can have a positive impact on motivation, user experience, and provide a significant contribution to the students' learning.
\end{abstract}

\section{KEYWORDS}

Design de Interface de Usuário, Design Thinking, Ensino de Computação, App Inventor, Ensino Fundamental

\section{INTRODUÇÃO}

$\mathrm{O}$ ensino do pensamento computacional tem sido o foco de esforços mundiais no contexto do ensino fundamental [14], no sentido de preparar os jovens para demandas atuais e futuras da sociedade [19, 27, 34]. Muitas dessas iniciativas se concentram no ensino de programação para apoiar as tarefas cognitivas envolvidas no pensamento computacional [27]. Nesse estágio educacional, a programação é tipicamente introduzida usando ambientes de programação baseados em blocos, como o App Inventor [22], para desenvolver aplicativos móveis.

No entanto, essas iniciativas geralmente não abrangem outras competências essenciais, como o design de interfaces de usuário (UIs - User Interfaces), essencial para a qualidade do software, e parte do corpo de conhecimento em computação [1]. O design da interface de usuário é um domínio que engloba princípios implícitos de organização, layout da tela, fluxos de trabalho, comportamentos interativos e linguagem visual [3]. Ele também inclui o design visual, responsável por expressar as potencialidades do sistema a partir de uma linguagem acessível ao usuário, que contribui significativamente para a usabilidade. Em razão disso, para a linguagem visual de uma interface conseguir atingir seu próposito, seus elementos (cores, tipografia e imagens) devem ser organizados levando em consideração os metaprincípios do design (consistência, hierarquia e personalidade) $[15,31]$.

As competências relacionadas ao design são importantes não apenas para os profissionais de tecnologia da informação (TI), mas para qualquer pessoa que se relacione com as habilidades do século XXI [2]. Diante da importância do tema, algumas diretrizes curriculares (como o K-12 Computer Science Framework [9]) indicam que o ensino de conceitos de design de interface deva ser inserido já no ensino fundamental. Isso porque a inserção do conhecimento de design de interface do usuário fornece vários benefícios e pode servir de base para sedimentar a cultura visual e as experiências de design [5], especialmente quando vinculadas à tecnologia. Além disso, as práticas relacionadas ao design estimulam a imaginação e buscam ensinar como tirar proveito dessa inventividade e colocá-la em prática [2]. Essas soluções e maneiras de se envolver com o mundo permitem que os indivíduos atuem como agentes de mudança e criação [15][27]. A estratégia de incorporar o ensino do design ao ensino de computação, permite também ampliar a percepção de que a computação é mais do que apenas codificar e, assim, aumentar o interesse nesse campo [4] e estimular a busca de uma carreira em TI [28][20]. Alguns países, como China e Coréia do Sul, estão seguindo essa premissa e começam a incorporar já alguns conceitos de design nas escolas [12][29].

Embora existam muitos esforços de design criativo (como o projeto City X [37] ou D.school [38] no contexto do movimento maker $^{l}$, existem poucas iniciativas específicas desenvolvidas para 
aprender conceitos fundamentais do design visual. As unidades instrucionais que abordam o design de interface geralmente ensinam diversos conceitos como design thinking, design de interface do usuário, design visual e/ou UX. No entanto, várias unidades de ensino estão limitadas a uma apresentação superficial de princípios gerais de interface, não orientando o aprendizado de aspectos mais concretos sobre como aplicar esses conceitos na prática, e em como programar elementos da interface do usuário em ambientes de programação baseados em blocos do que princípios de design [41]. Além disso, focar apenas nos "fundamentos do design" ou na "programação da interface do usuário", que não abrangem aplicativos do mundo real, pode ser uma abordagem limitada por não conectar-se aos interesses e vidas pessoais específicos dos alunos. Outra deficiência está relacionada à ausência de modelos de avaliação que mensurem o aprendizado dos alunos em relação a essas competências. Embora existam vários artigos que apresentam avaliações baseadas nos artefatos criados [8][24][34], a maioria desses estudos são limitados a apenas um critério de avaliação para aferir habilidades de design da interface de usuário. Isso mostra a necessidade de se criar unidades instrucionais mais detalhadas, que utilizem abordagens práticas e que possam, efetivamente, ajudar os alunos a aprender como desenvolver aplicativos do mundo real.

Assim, a fim de explorar a questão do aprendizado de design de interface do usuário no ensino fundamental, apresentamos os resultados de pesquisa sobre desenvolvimento, aplicação e avaliação de uma unidade instrucional criada com o objetivo de ensinar competências básicas de design visual como parte do ensino da computação, propondo o desenvolvimento de aplicativos móveis com o App Inventor.

\section{Metodologia da pesquisa}

O presente trabalho consiste em uma pesquisa exploratória utilizando uma abordagem de método misto, conforme ilustrado na Figura 1.

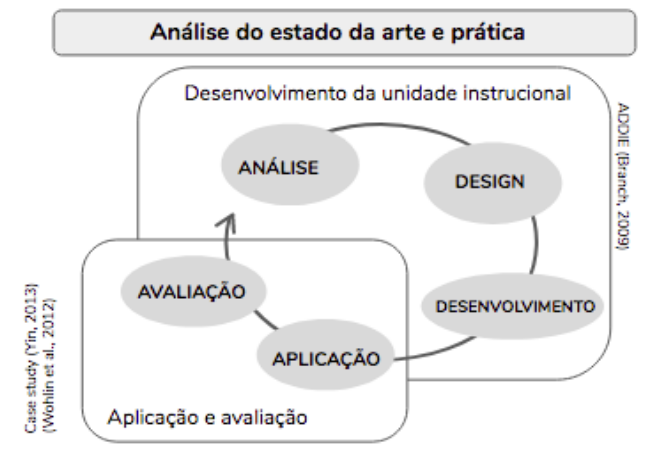

Figura 1: Metodologia da Pesquisa (elaborada pelos autores).

Análise do estado da arte e prática. Inicialmente, foi realizando um estudo de mapeamento sistemático, seguindo Petersen et al. [17], a fim de revisar a literatura existente sobre unidades instrucionais para o ensino do design da interface de usuário no ensino fundamental. Revisamos artigos publicados em inglês e português disponíveis na web por meio das principais bibliotecas e bancos de dados digitais (IEEE Xplore, ACM Digital
Library, Wiley, Springer e Scopus), Google Scholar, além de cursos em plataformas MOOC, incluindo Udemy, Edx, Khanacademy e Coursera (para minimizar os riscos de omissão). Os resultados desta revisão são apresentados em [41].

Desenvolvimento de unidade instrucional. A unidade instrucional "Faça o seu primeiro aplicativo" foi sistematicamente desenvolvida, seguindo o modelo de design instrucional ADDIE [26], incorporando o ensino das competências de design visual. $\mathrm{Na}$ primeira etapa, foi analisado o contexto educacional e foram definidos os objetivos de aprendizagem. Com base nessa análise foi desenvolvida a estratégia instrucional e o material instrucional (adotando o App Inventor como um ambiente de programação baseado em blocos).

Aplicação e avaliação da unidade instrucional. A unidade instrucional foi aplicada e avaliada por meio de um estudo de caso exploratório em uma escola pública da cidade de Florianópolis/SC no primeiro semestre de 2019. O estudo de caso foi sistematicamente definido, planejado, executado e analisado seguindo o processo proposto por Wohlin et al. [7] e Yin [14]. A avaliação foi definida usando a abordagem Goal/Question/Metric (GQM) [33] usando os modelos de avaliação dETECT [5] e MEEGA+ [12]. Os dados foram coletados por meio de vários instrumentos (questionários, observação e análise de artefatos desenvolvidos) e analisados de forma descritiva.

\section{Desenvolvimento da Unidade Instrucional "Faça seu primeiro aplicativo"}

\subsection{Análise de contexto}

O público-alvo desta unidade de ensino são alunos, com idades entre 9 e 15 anos, do ensino fundamental. Normalmente, a maioria dos estudantes nessa idade já possui conhecimentos e habilidades no uso de computadores e outros dispositivos. A maioria deles possui telefone celular próprio (predominantemente Android) e costuma passar muito tempo online, principalmente em redes sociais, vídeos, músicas e/ou jogos digitais [35] [36]. Os estudantes em geral já utilizam softwares, mas poucos sabem como criar artefatos computacionais. Normalmente, os alunos dessa faixa etária não têm nenhuma competência específica de design, aprendida apenas de maneira indireta como parte das aulas de arte. Quanto ao ensino de práticas de computação para essa faixa etária, geralmente ocorrem de maneira multidisciplinar, em aulas regulares ou como atividades extracurriculares. No entanto, as unidades instrucionais que abordam esse conteúdo geralmente têm uma duração relativamente curta devido a restrições de tempo do currículo escolar. As aulas geralmente ocorrem em laboratórios de informática com computadores (ou laptops) e acesso à internet. As turmas normalmente têm uma média de 25 a 40 alunos. Especificamente, no contexto das escolas públicas, pode haver poucos recursos disponíveis para a aquisição de material instrucional. 


\subsection{Objetivos de Aprendizagem}

O objetivo da unidade instrucional "Faça seu primeiro aplicativo" é ensinar competências visuais de design de interface de usuário integradas ao ensino de computação. Os objetivos de aprendizagem são derivados das principais diretrizes curriculares [1][2][9] e da teoria do design visual [3][15][31] para o contexto específico no ensino fundamental (Quadro 1).

\section{Quadro 1: Objetivos de aprendizagem}

\begin{tabular}{|l|l|l|}
\hline ID & Objetivo de aprendizagem & Área de conhecimento \\
\hline OA1 & $\begin{array}{l}\text { Entender os algoritmos como um conjunto } \\
\text { de instruções passo a passo para realizar } \\
\text { tarefas. }\end{array}$ & $\begin{array}{l}\text { Algoritmos e } \\
\text { Programação }\end{array}$ \\
\hline OA2 & $\begin{array}{l}\text { Criar sistemas de software interativos que } \\
\text { incluem eventos, condicionais, variáveis, } \\
\text { listas e sequências de caracteres usando uma } \\
\text { linguagem de programação visual baseada } \\
\text { em blocos. }\end{array}$ & $\begin{array}{l}\text { Algoritmos e } \\
\text { Programação }\end{array}$ \\
\hline OA3 & $\begin{array}{l}\text { Criar o design visual (cor, tipografia, imagem } \\
\text { e composição) do sistema de software } \\
\text { interativo. }\end{array}$ & $\begin{array}{l}\text { Design visual, } \\
\text { Algoritmos e } \\
\text { Programação }\end{array}$ \\
\hline OA4 & $\begin{array}{l}\text { Testar e refinir um sistema de software } \\
\text { interativo com relação à funcionalidade e } \\
\text { usabilidade. }\end{array}$ & $\begin{array}{l}\text { Algoritmos, } \\
\text { Programação e } \\
\text { Engenharia de software }\end{array}$ \\
\hline OA5 & $\begin{array}{l}\text { Compartilhar o sistema de software } \\
\text { interativo que foi desenvolvido }\end{array}$ & Comunicação \\
\hline
\end{tabular}

\subsection{Estratégia Instrucional}

Com base na análise de contexto, a unidade instrucional foi projetada como um curso composto por 6 aulas, cada uma com duração de 1,5 horas. O curso foi desenvolvido para ser aplicado de forma presencial, suportado por uma plataforma de aprendizagem (Moodle).

Adotamos uma abordagem de ação computacional [23] com uma estrutura para o ensino de computação socialmente relevante, buscando oferecer aos alunos a oportunidades de criar soluções de computação que tenham impacto direto em suas vidas e na comunidade. A unidade instrucional é integrada ao ensino em computação de maneira interdisciplinar, como parte das aulas de ciências, para desenvolver a conscientização ambiental, com foco no tópico de sustentabilidade e reciclagem. Nesse contexto, a unidade se concentra na criação de um aplicativo móvel para visualizar os pontos de coleta da ECOPET, uma ONG local que organiza a coleta de tampas de garrafas de plástico que são vendidas para fins de reciclagem, no intuíto de usar os recursos obtidos para pagar a castração de animais abandonados.

Vários métodos instrucionais são adotados com foco predominantemente no aprendizado ativo, propondo atividades práticas de programação e de design, visando permitir que os alunos, em pares, pratiquem e explorem os conceitos ensinados $[14,27,30]$. Para ilustrar e tornar a aprendizado do conceito de algoritmos mais lúdico também foi adotado um jogo educacional [5] e o ensino do design visual foi reforçado por meio de vários exercícios "desplugados".

\section{Quadro 2: Conteúdo programático do curso}

\begin{tabular}{|l|l|l|}
\hline Aula & Conteúdo & $\begin{array}{l}\text { Objetivo de } \\
\text { aprendizagem }\end{array}$ \\
\hline Medição inicial & $\begin{array}{l}\text { Motivando computação e conceitos básicos } \\
\text { (algoritmos) }\end{array}$ & OA1 \\
\hline 1 & $\begin{array}{l}\text { Introdução à programação com o App Inventor - } \\
\text { EcoPET }\end{array}$ & OA2 \\
\hline 2
\end{tabular}

\begin{tabular}{|l|l|l|}
\hline \multicolumn{3}{|l|}{ Medição de pré-teste de design visual } \\
\hline 3 & $\begin{array}{l}\text { Criação e teste de design visual com o App } \\
\text { Inventor (cores e tipografia) }\end{array}$ & OA2, OA4 \\
\hline 4 & $\begin{array}{l}\text { Criação e teste de design visual no App Inventor } \\
\text { (imagem e composição) }\end{array}$ & OA3, OA4 \\
\hline Medição de pós-teste de design visual \\
\hline 5 & $\begin{array}{l}\text { Ensinar a apresentar o trabalho e compartilhar o } \\
\text { aplicativo }\end{array}$ & OA5 \\
\hline 6 & Apresentação dos aplicativos criados pelos alunos & OA5 \\
\hline \multicolumn{2}{|l|}{ Medição final } \\
\hline
\end{tabular}

Concentrando-se inicialmente no aprendizado de conceitos básicos de programação (eventos, strings, variáveis e mapas), os alunos aprenderam, já na segunda aula, a desenvolver uma versão simples em wireframe do aplicativo EcoPET. Na terceira aula, em razão da curta duração do curso, os alunos receberam uma versão completa em wireframe do aplicativo (incluindo armazenamento de localizações, sensores, etc.) para desenvolver o design visual dos seus aplicativos.

Adotamos o App Inventor [22] como ambiente de programação baseado em blocos para o desenvolvimento de aplicativos móveis open source. Este ambiente de programação é de fácil utilização, desenvolvido para ser utilizado pela faixaetária alvo do curso, e já utilizado por mais de 400 mil usuários únicos por mês localizados em 195 países.

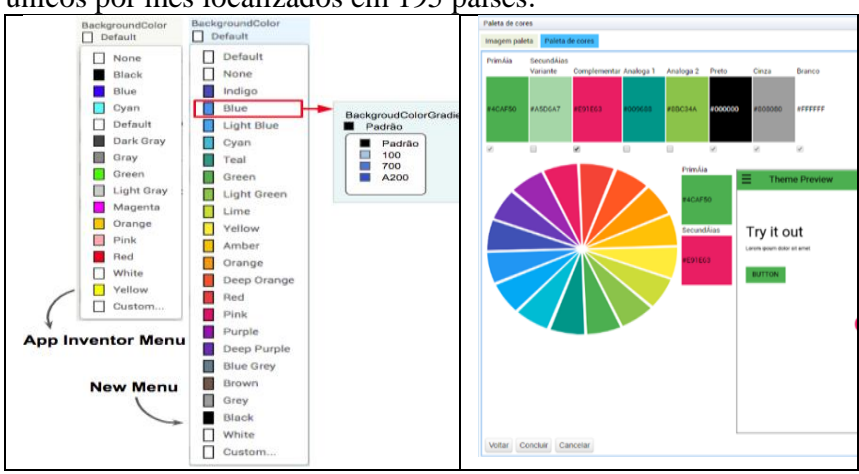

Figura 2: Exemplos de funcionalidades de design de interface do usuário implementado no App Inventor [40]

Para este curso foram modificadas algumas funcionalidades da versão original App Inventor do MIT, com o propósito de deixá-lo em conformidade com os guias de estilo do Android (como por exemplo: o menu de cores e o catálogo de ícones) e foram implementadas novas funcionalidades para orientar o aluno no processo de seleção de cores e, desta forma, contribuir para o ensino do design da interface do usuário (Figura 2). Além disso, para atender à estratégia instrucional, vários materiais instrucionais foram desenvolvidos em português (Quadro 3).

\section{Quadro 3: Exemplos de materiais instrucionais desenvolvidos}

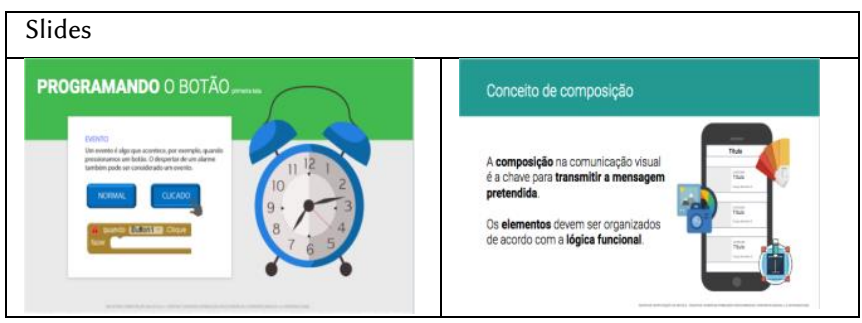




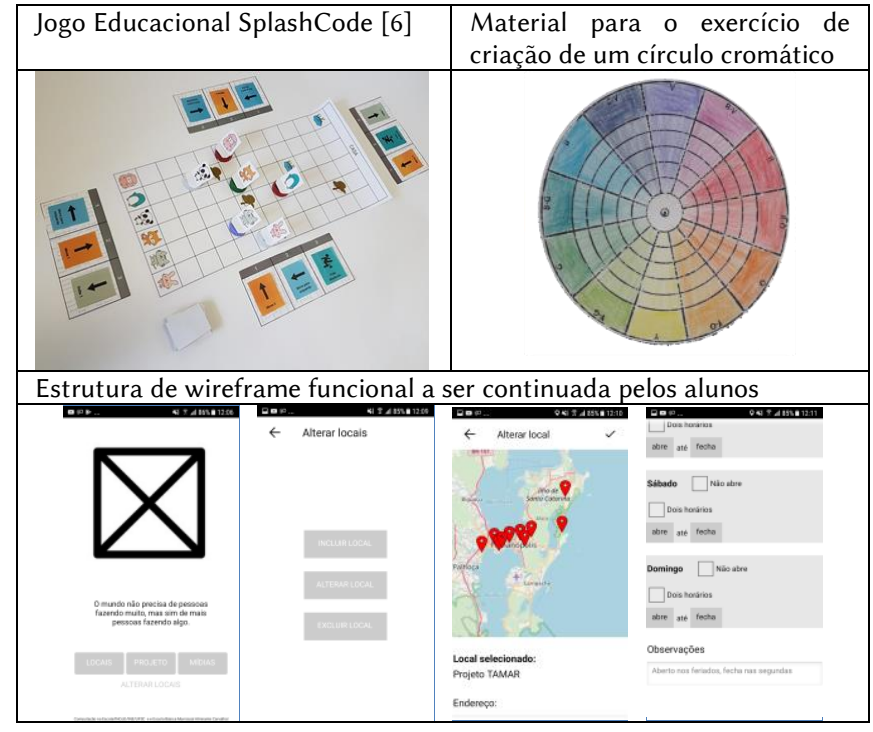

Com a intenção de aferir a aprendizagem dos alunos, são adotadas diferentes formas de avaliação baseadas nos objetivos de aprendizagem. Para avaliar o design visual (OA3 e OA4), baseado no desempenho do artefato criado pelos alunos, foi definida uma rubrica específica com níveis de desempenho numa escala ordinal de 5 pontos, variando de totalmente alcançado a não alcançado (Quadro 4). A avaliação também é suportada pela ferramenta CodeMaster 2.0 [34], que fornece uma avaliação automática do design visual dos projetos do App Inventor em conformidade com a teoria e nas diretrizes do design visual.

Quadro 4: Rubrica da avaliação baseada no desempenho do design visual das interfaces de usuário criadas

\begin{tabular}{|l|}
\hline $\begin{array}{l}\text { Em relação ao design visual do aplicativo criado pelo (s) aluno } \\
\text { (s): }\end{array}$ \\
\hline A paleta de cores é consistente com o tema escolhido. \\
\hline A organização das cores facilita a interação do usuário. \\
\hline As cores auxiliam na hierarquia de informações. \\
\hline $\begin{array}{l}\text { O contraste entre a cor do texto e do fundo da tela asseguram a } \\
\text { legibilidade. }\end{array}$ \\
\hline Os textos apresentam tamanho de fonte agradáveis para leitura \\
\hline $\begin{array}{l}\text { O alinhamento do texto contribui para leitura e harmonia do design } \\
\text { visual. }\end{array}$ \\
\hline O tamanho das fontes auxilia na hierarquia de informações. \\
\hline As imagens estão de acordo com o tema. \\
\hline As imagens estão nítidas (não estão pixeladas e distorcidas). \\
\hline Os ícones são intuitivos (fáceis de interpretar). \\
\hline $\begin{array}{l}\text { As telas respeitam o mesmo padrão em relação aos elementos de cor, } \\
\text { imagem e tipografia (tamanho, família, estilo, peso). }\end{array}$ \\
\hline De modo geral o design visual está agradável e organizado. \\
\hline
\end{tabular}

\section{Aplicação em uma escola pública}

O curso foi aplicado e avaliado com 24 alunos de uma escola pública, Escola Básica Municipal Almirante Carvalhal, em Florianópolis/SC, como parte das aulas de ciências, durante o primeiro semestre de 2019. Onze meninas e treze meninos do $5^{\circ}$ ano (9 a 11 anos) participaram da aplicação.
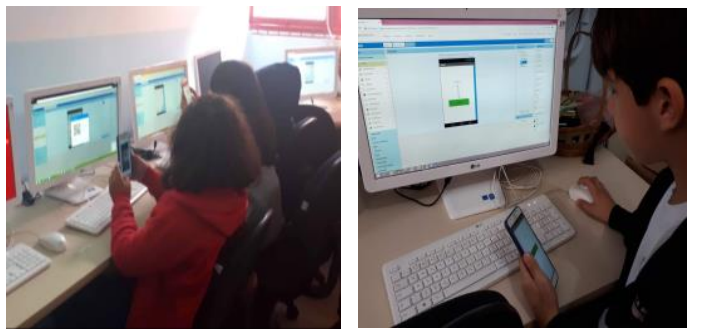

Figura 3: Alunos durante atividades de design visual

No início, buscou-se a motivação sobre a área de computação a partir da introdução de conceitos básicos como algoritmos. Para complementar a compreensão sobre algoritmos os alunos jogaram o jogo SplashCode [6]. Durante a segunda aula, seguindo passo a passo um tutorial com a ajuda de instrutores, os alunos aprenderam a programar uma versão simples em wireframe do aplicativo EcoPET. Na aula seguinte os alunos receberam uma versão completa em wireframe do aplicativo EcoPET para criar o design visual. Foram ensinados conceitos básicos de design e os alunos realizaram atividades práticas (em papel). Nessas aulas os alunos também puderam trabalhar em seus projetos, criando e personalizando os elementos da interface dos seus aplicativos com o App Inventor.

Após a finalização do design visual da interface dos aplicativos, os alunos receberam orientações de como apresentar e compartilhar seus projetos. No último encontro foi realizado um evento na escola para apresentação dos aplicativos que contou com a presença de pais, outros alunos e representantes da ONG (Figura 4).

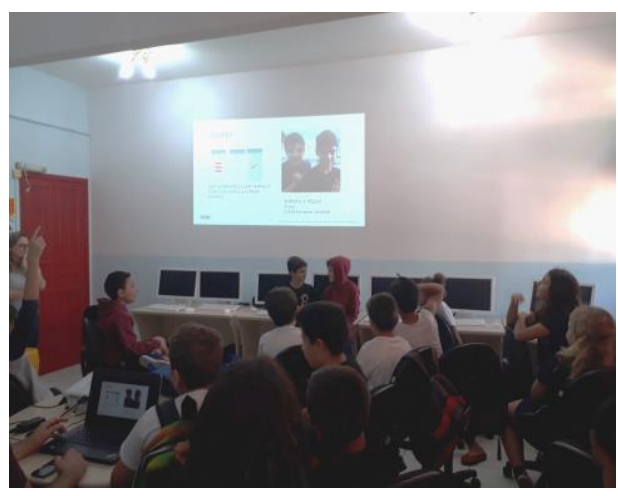

Figura 4: Alunos Apresentando seus Resultados

\section{Avaliação da Unidade Instrucional}

\subsection{Definição da Avaliação}

Este estudo teve como objetivo analisar se o curso desenvolvido alcança os objetivos de aprendizagem (especialmente OA3 e OA4) e se o modo de apresentação da estratégia instrucional fornece uma experiência de aprendizado motivadora e envolvente. Para tanto, a unidade instrucional foi 
avaliada por meio de um estudo de caso exploratório, utilizando um desenho de pesquisa pós-teste one-shot, que permite a pesquisa aprofundada de um indivíduo, grupo ou evento [8][26]. Com base nos modelos dETECT [5] e MEEGA+ [11], o objetivo da avaliação foi decomposto em dois fatores de qualidade e suas dimensões (Figura 5). A experiência de aprendizagem foi definida como um fator de qualidade que abrange o envolvimento dos alunos na atividade de aprendizagem, incluindo sentimentos, prazeres e interações [11][16]. O aprendizado foi medido por meio das percepções dos alunos [11][10] e também com base no desempenho real.

A coleta de dados foi operacionalizada por meio de uma avaliação baseada no desempenho e de um questionário de autoavaliação respondido pelos alunos, a fim de coletar dados sobre suas percepções da unidade instrucional.

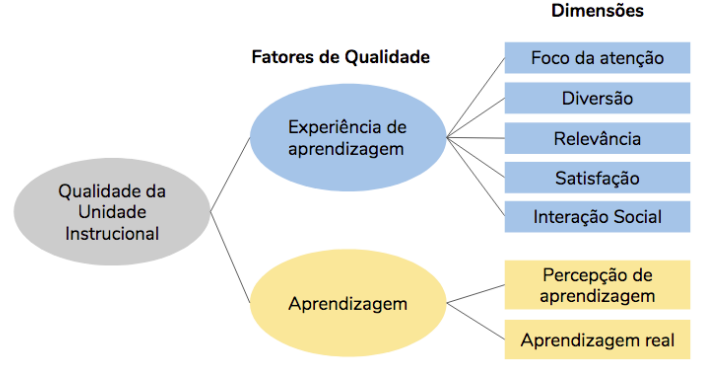

Figura 5: Decomposição dos fatores de qualidade avaliados (elaborado pelos autores).

\subsection{Análise de dados}

5.2.1 Avaliação da experiência de aprendizagem. Vinte e um alunos avaliaram a experiência de aprendizado como positiva e consideraram o curso divertido (Figura 6). Desses, dezessete alunos indicaram as aulas e o contéudo de design visual como divertidos e/ou muito divertidos. O único tópico considerado muito chato por um aluno foi o "programação e testes". Além disso, vale destacar que dezenove alunos consideraram que o tempo das aulas passou rápido ou muito rápido.

Foi possível perceber ainda que a maioria dos estudantes considerou os tópicos ensinados fáceis de aprender, poucos reconheceram dificuldades na programação e testes e design visual (Figura 6).

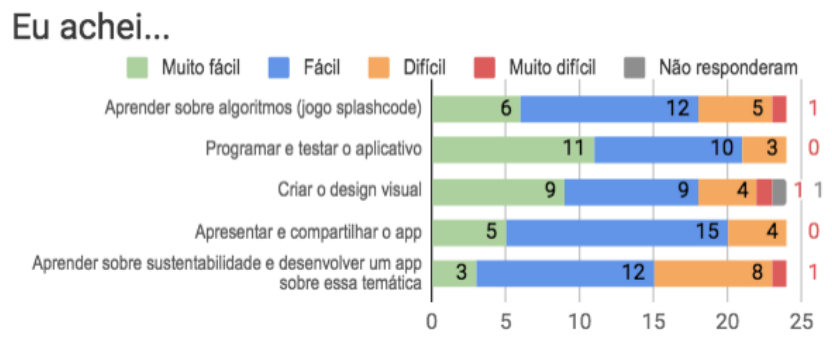

Figura 6. Frequência de respostas em relação à percepção da facilidade de aprendizado
Dezoito dos vinte e quatro estudantes manifestaram satisfação e disseram que se sentiram à vontade em desenvolver seus aplicativos com um colega, os demais preferiram fazer a atividade sozinhos. Os alunos também gostaram de apresentar e compartilhar os seus resultados. Ao final, eles demonstraram interesse em aprender mais sobre o conteúdo do curso, especialmente no desenvolvimento de aplicativos (14 alunos) e design visual (10 alunos).

5.2.2 Avaliação de aprendizagem. Para avaliar o aprendizado dos objetivos OA3 e OA4 foram utilizados os resultados da avaliação baseada no desempenho do design visual dos aplicativos criados pelos alunos, adotando a rubrica apresentada no Quadro 4 (Figura 7).

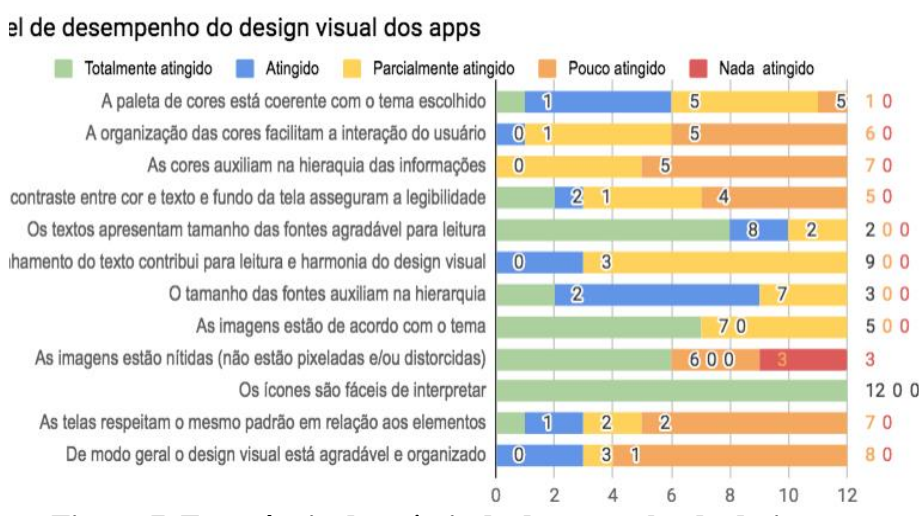

Figura 7. Frequência dos níveis de desempenho do design visual

A partir da análise dos artefatos, observamos que os alunos tinham dificuldade em aplicar os meta-princípios de design, pois usaram cores saturadas e um excesso de elementos que prejudicaram a personalidade, a hierarquia e a composição das suas interfaces. Eles também adotaram suas preferências pessoais em vez de escolher cores de maneira harmoniosa, considerando o tema do aplicativo. Além disso, os alunos demonstraram dificuldades em dimensionar imagens e/ou selecionar imagens em harmonia com a paleta de cores escolhida. No que diz respeito aos elementos de texto, os aplicativos expressavam hierarquia, mas em alguns casos foram usados formatos não recomendados, como alinhamento centralizado e execsso de fontes em itálico. No entanto, quando comparado a aplicativos escolhidos aleatoriamente na App Inventor Gallery (Figura 8), pode-se inferir que os designs visuais criados pelos alunos deste curso (Figura 9), mesmo considerando todos os pontos negativos, apresentam interfaces com design visual esteticamente mais atraentes. 


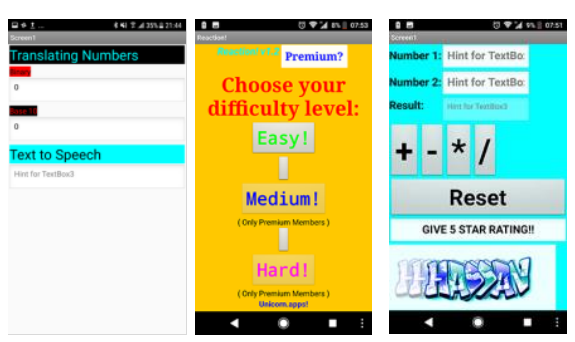

Figura 8. Interfaces de aplicativos selecionados aleatoriamente na App Inventor Gallery

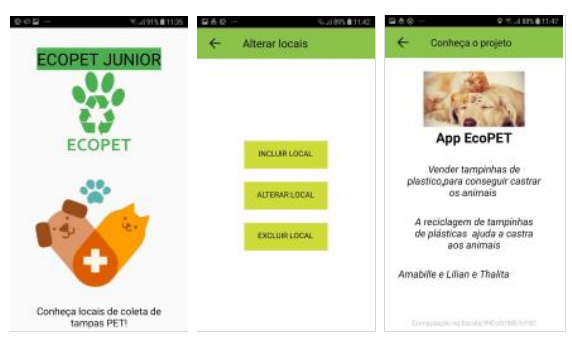

Figura 9. Exemplos do design visual criado pelos alunos

\section{Discussão}

Os resultados mostram que a maioria dos alunos gostou da unidade instrucional. Observamos que os alunos participaram ativamente, motivados e ansiosos para explorar as funcionalidades do App Inventor e para criar e testar seus aplicativos. Um destaque do ambiente do App Inventor é certamente a possibilidade de realizar testes em tempo real, visualizando o aplicativo em execução no smartphone, pois contribuiu significativamente para a motivação dos alunos. Também os ajudou a detectar erros e melhorar o aplicativo. Além disso, observamos que o aprimoramento do App Inventor, no que concerne ao suporte adicional do design visual em conformidade com as diretrizes do Android, tornou o processo de design mais eficiente e eficaz, contribuindo para o desenvolvimento de aplicativos visualmente mais atraentes. Notou-se que, ao criar um aplicativo funcional, os alunos também perceberam que são capazes de criar artefatos de software que podem contribuir para suas vidas e para a comunidade. Os alunos estavam ansiosos para mostrar seus aplicativos para seus amigos e familiares. Muitos estudantes também expressaram o desejo de desenvolver outras aplicativos de música e jogos, indicando que o curso os motivou a explorar a computação.

No entanto, também observamos algumas dificuldades durante a aplicação que podem estar relacionadas a esse nível educacional, quando comparado às nossas experiências de aplicação desse tipo de curso em grupos menores de alunos e em níveis educacionais mais avançados. Uma questão é que os alunos do $5^{\circ}$ ano priorizaram suas próprias preferências (por exemplo, com relação às cores ou formatos dos botões) em vez de selecioná-las de acordo com o tema do aplicativo e às diretrizes de design da interface de usuário. Essas questões também levaram a discussões entre os pares de estudantes que trabalharam em conjunto, quando eles tinham preferências conflitantes. Embora os alunos demonstrassem habilidades para resolver conflitos, geralmente usando os dois estilos visuais no mesmo aplicativo, essas escolhas refletiram em inconsistências em relação ao design visual. Observamos também que os alunos desse curso possuiam um perfil mais disperso, provavelmente devido à faixa-etária e agravado pelo limitado espaço físico do laboratório. Ainda que os alunos tenham concluído seus projetos durante a duração da unidade instrucional, acredita-se que uma duração mais longa contribuiria mais positivamente para a qualidade dos artefatos criados, pois forneceria mais tempo para os alunos aprenderem sobre a linguagem visual. No entanto, em geral, a avaliação do curso proporcionou resultados promissores em termos de experiência e aprendizado, motivando novas aplicações em uma escala maior deste curso no ensino fundamental.

Ameaças à validade. $\mathrm{O}$ desenho do estudo de caso aplicado nesta pesquisa pode causar algumas ameaças quanto à validade dos resultados. Uma ameaça é a falta de conhecimento dos alunos no início, o que torna impossível comparar o resultado no final do curso. Isso é ainda mais evidente quando não há grupo controle para comparar os efeitos identificados. No entanto, neste estudo, optamos por realizar um estudo de caso em vez de um experimento para limitar as interrupções ao fluxo normal das aulas. $\mathrm{O}$ fato do curso ter sido aplicado a apenas uma turma de uma escola reduz a possibilidade de generalização dos resultados. Outra ameaça possível é que aspectos como diversão e satisfação são difíceis de medir e são capturados por medidas subjetivas. Para contornar isso, derivamos os instrumentos de medição sistematicamente com base nos modelos existentes, como o MEEGA+, que já foi avaliado com relação à sua confiabilidade e validade em larga escala [12].

\section{Conclusão}

Este artigo apresenta o desenvolvimento, aplicação e avaliação de uma unidade instrucional com o objetivo de integrar o ensino de design de interface visual ao ensino de computação. Os resultados de uma primeira aplicação fornecem uma indicação inicial de que os conceitos de computação e design podem ser introduzidos de maneira multidisciplinar no ensino fundamental e podem contribuir positivamente para o aprendizado de habilidades além da computação básica. Os alunos avaliaram a experiência da unidade instrucional de maneira positiva, mostrando que esses conceitos podem ser ensinados de maneira divertida, eficiente e eficaz. A unidade instrucional também deixou os alunos interessados em aprender mais sobre computação e, principalmente, design visual. Usando o App Inventor, os alunos puderam desenvolver aplicativos totalmente funcionais para contribuir em suas comunidades. $\mathrm{O}$ aprimoramento do suporte ao design de interface de usuário no App Inventor favoreceu o desenvolvimento do design visual. Essa facilidade percebida de programação e design também os motivou a desenvolver outros aplicativos. Devido a esse resultado positivo, atualmente estamos refinando os materiais instrucionais e planejando a aplicação do curso em uma escala maior. 


\section{REFERÊNCIAS}

[1] ACM/IEEE. 2013. Computer Science Curricula 2013: Curriculum Guidelines for Undergraduate Degree Programs in Computer Science. Nova York, NY.

[2] AIGA. 2013. The professional association for design, https://www.aiga.org

[3] A. Cooper, R. Reimann, and H. Dubberlyl, 2014. About face: the essentials of interaction design. Indianápolis: John Wiley and Sons.

[4] A. Robinson, M. A. Pérez-Quinones and G. Scales. 2015. Understanding the attitudes of African American middle school girls toward computer science. In: Proc. of Research in Equity and Sustained Participation in Engineering, Computing, and Technology, Charlotte, NC.

[5] C. G. von Wangenheim, G. Petri, A. Zibetti, A. F. Borgatto, J. C. Hauck, F. Pacheco, and R. M. Filho. 2017. DETECT: A Model for the Evaluation of Instructional Units for Teaching Computing in Middle School. Informatics in Education, 16(2), 301-318.

[6] C. G. von Wangenheim, G. A. S. Medeiros, R. M. Filho, G. P., F.C. Pinheiro, M. N. F. Ferreira, and J. C. Hauck. 2019. SplashCode - A Board Game for Learning an Understanding of Algorithms in Middle School. Informatics in Education, accepted for publication.

[7] C. Wohlin, P. Runeson, M. Host, M. C. Ohlsson, B. Regnell and A. Wesslén. 2012. Experimentation in Software Engineering. Berlin: Springer.

[8] CODE.ORG. 2018. Code.org. https://curriculum.code.org/csd-1718/unit4/

[9] CSTA. 2017. K-12 Computer Science Framework.

[10] Daniel L. Moody and Guttorm Sindre. 2003. Evaluating the Effectiveness of Learning Interventions: an Information Systems Case Study. In: Proc. of the 11th European Conf. on Information Systems, Naples, Italy.

[11] G. Petri, C. G. von Wangenheim and A. F. Borgatto. 2018. MEEGA+, Systematic Model to Evaluate Educational Games. In: Lee N. (Ed.), Encyclopedia of Computer Graphics and Games. Springer.

[12] Imogen West-Knights. 2017. Why are schools in China looking west for lessons in creativity? Financial Times.

[13] ISO/IEC 9241-210. (2010). Ergonomics of human-system interaction-part 220: Processes for enabling, executing and assessing human-centered design within organizations. International Standardization Organization (ISO).

[14] J. M. Wing. 2006. Computational thinking. Communications of the ACM, 49(3), 33-35.

[15] J. J. Garret. 2011. The Elements of User Experience: User-Centered Design for the Web and Beyond, (2nd ed.). Berkeley: New Riders.

[16] J. M. Keller. 1987. Development and Use of the ARCS Model of motivational Design. Journal of Instructional Development, 10(3), 2-10.

[17] K. Petersen, R. Feldt, S. Mujtaba, and M. Mattsson. 2008. Systematic mapping studies in Software engineering. In: Proc. of the 12th Int. Conf. on Evaluation and Assessment in Software Engineering, Swindon, UK.

[18] K. S. Christensen, M. Hjorth, and O. S. Iversenl. 2016. Towards a formal assessment of design literacy: Analyzing K-12 students' stance towards inquiry. Design Studies, 46, 125-151.

[19] M. Resnick et al. Scratch: Programming for all. Communications of the ACM, 52(11), 60-67.

[20] M. Craig and D. Horton. 2009. Gr8 designs for Gr8 girls: a middle-school program and its evaluation. ACM SIGCSE Bulletin. 41(1), 221-225.

[21] M. N. F. Ferreira, F. C. Pinheiro, R. M. Filho, C. G. von Wangenheim and B. S. Gonçalves. 2018. Material supplemental - Ensinando Design de Interface de Usuário na Educação Básica: Um Mapeamento Sistemático do Estado da Arte e Prática. Relatório Técnico, INCoD/INE/UFSC, Florianópolis/SC.

[22] MIT. 2019. App Inventor. http://appinventor.mit.edu/explorel.

[23] M. Tissenbaum, M. Sherman, J. Sheldon e H. Abelson. 2019. From Computational Thinking to Computational Action. ACM Interactions, 62(3), 34-36.

[24] P. Chen and R. Huang. 2017. Design thinking in App inventor game design and development: A case study. In: Proc. of 17th Int. Conf. on Advanced Learning Technologies. Timisoara, Romania, 139-141.

[25] R. K. Yin. 2017. Case study research: design and methods. Thousand Oaks: Sage Publications, Inc.

[26] R. M. Branch. 2009. Instructional design: The ADDIE approach, (2nd ed.). New York: Springer.

[27] S. Grover and R. Pea. 2013. Computational thinking in K-12: A review of the state of the field. Educational Researcher, 42(1), 38-43.

[28] S. Yardi, P. Krolikowski, T. Marshall, and A. Bruckman. 2008. An IHC approach to computing in the real world. Journal on Educational Resources in Computing, 8(3), 9.

[29] S. Ahn. (2014). A Study on Information Science Curriculum of Productivity Tools to Increase Ability for Problem Solving in Elementary and Middle School. Journal of Korea Association of Information Education, 18(2), 235-242.

[30] S. Y. Lye and J. H. Ling. 2014. Review on teaching and learning of computational thinking through programming: What is next for $\mathrm{K}-12$ ? Computers in Human Behavior, 41(C), 51-61.

[31] T. Schlatter and D. Levinson. 2013. Visual usability: principles and practices for designing digital applications. Morgan Kaufman.
[32] UXQB. 2018. CPUX-F Curriculum and Glossary. https://uxqb.org/wpcontent/uploads/documents/CPUX-F_EN_Curriculum-and-Glossary.pdf.

[33] V. R. Basili, G. Caldiera, and H. D. Rombach (1994). Goal Question Metric Paradigm. In J. J. Marciniak, Encyclopedia of Software Engineering, New York: Wiley-Interscience.

[34] I. S. Solecki, J. V. A. Porto, K. A. Justen, C. G. von Wangenheim, N. C. Alves, A. F. Borgatto and J. C. R. Hauck. 2019. CodeMaster UI Design - App Inventor: A Rubric for the Assessment of the Interface Design of Android Apps developed with App Inventor. In: Proc. of the 18th Brazilian Symposium on Human Factors in Computing Systems, Vitória, Brazil.

[35] Statcounter Global Stats. 2019. Mobile Operating System Market Share Brazil. https://gs.statcounter.com/os-market-share/mobile/brazil.

[36] TIC Kids Online Brasil. 2018. https://www.cetic.br/tics/kidsonline/2018/ criancas/A1/.

[37] City x Project. 2018. http://www.cityxproject.com

$\begin{array}{ccc}\text { [38] Stanford } & \text { University. } & \text { D.scholl. }\end{array}$ https://dschool.stanford.edu/programs/k12-lab-network.

[39] R. Samagaia, and D. D. Neto. (2015). Educação científica informal no movimento "Maker". In: 10th Encontro Nacional de Pesquisa em Educação em Ciências. Águas de Lindóia, São Paulo.

[40] Pinheiro, F., Gresse Von Wangenheim, C., Hauck, J. C. R., Ferreira, M. N. F. Evolução da Ferramenta App Inventor para Suportar o Ensino de Design Interface na Educação Básica. Anais do Workshop de Informática na Escola no Congresso Brasileiro de Informática da Educação, Brasília/DF, 2019.

[41] Ferreira, M. N. F., Pinheiro, F., Missfeldt Filho, R. , Gresse Von Wangenheim, C. Ensinando Design de Interface de Usuário na Educação Básica: Um Mapeamento Sistemático do Estado da Arte e Prática. Anais do Workshop de Informática na Escola no Congresso Brasileiro de Informática da Educação, Brasília/DF, 2019 